\title{
An Algorithm for the Solution of Certain Differential-Difference Equations of Advanced Type
}

\author{
By G. Greaves
}

Abstract. The differential-difference problem

$$
\frac{d}{d s}\{s J(s)\}+J(s+1)=0 \quad(\operatorname{Re}(s)>0) ; \quad s J(s) \rightarrow 1 \quad \text { as } s \rightarrow 0,
$$

which arises in the theory of numbers, is studied and an algorithm developed for the simultaneous numerical approximation of the derivatives $J^{(n)}(s)$. An error bound is established for the process described and its numerical performance and stability are discussed.

1. Introduction. The solution,

$$
J(s)=\int_{0}^{\infty} e^{-s x} \exp \left\{-\int_{x}^{\infty} \frac{e^{-t}}{t} d t\right\} d x,
$$

of the differential-difference problem

$$
\begin{gathered}
\frac{d}{d s}\{s J(s)\}+J(s+1)=0, \\
s J(s) \rightarrow 1 \text { as } s \rightarrow 0+,
\end{gathered}
$$

where $J(s)$ is analytic in the half-plane $\operatorname{Re}(s)>0$, is of some importance in the theory of numbers; see, for example, [2], [5]. In particular the present author needed, in connection with the work described in [5], numerical approximations to the first few derivatives $J^{(n)}(1)$. In this paper we discuss an algorithm (which has been implemented by the author on an ICL 2980 computer) which provides simultaneous approximations to these numbers. The algorithm is based upon the differential-difference equation (1.2) rather than upon the integral representation (1.1). We establish error bounds (see (3.3), (4.2) below) for the processes we describe.

The method applies at least to certain other equations

$$
\frac{d}{d s}\{s g(s)\}=a g(s)+b g(s+1)
$$

of importance in this subject: see [6], [7]. The present discussion (see (2.1) below) deals simultaneously with the cases $b=-1, a$ a negative integer; the author has implemented a similar algorithm relating to the case $b=+1$.

The algorithm involves a representation of the solution $J(s)$ which, for some purposes (e.g., the application in [5]), is more convenient than the integral representation (1.1). We express $J(s)$ in terms of a series of $I+1$ functions $\psi_{i}(0 \leqslant i \leqslant I)$, of which $\psi_{i}$ removes a singularity at the integer $-i$. There remains the solution $f_{i}$ of

Received December 30, 1980; revised April 1, 1981.

1980 Mathematics Subject Classification. Primary 65R20, 45L10. 
a residual differential-difference equation; we approximate to the derivatives $f_{i}^{(n)}(s)$ for $0 \leqslant n \leqslant N$ by a rather straightforward process involving the solution of a triangular system of $N+1$ linear algebraic equations. It appears that, of these two processes, the first, if used in isolation, fails to yield convergent approximations (as $I \rightarrow \infty)$ to $J^{(n)}(1)$ in the case $n=0$, while, for any $I$, the second process is not convergent as $N \rightarrow \infty$. We will show, however, that when used in the appropriate combination very satisfactory convergence properties result.

The applications referred to above of the solutions of "advanced" equations, such as (1.2), have a characteristic feature. This is that they are used to obtain information about solutions of "retarded" equations such as (6.2) below. In contrast to (1.1), these solutions are not analytic at any positive integer. As an illustration of this feature we show that the number

$$
J(1)=0.62432998854355087099 \ldots
$$

is identical to a constant computed many years ago by Dickman [4].

For a general reference on the subject of differential-difference equations the reader may consult [1].

2. Removal of Singularities. Observe first (there are details in [5]) that the function $J$, as defined by (1.1), has the property that $J(s)-1 / s$ has an analytic continuation into $\operatorname{Re}(s)>-1$, the singularity at 0 being removable.

By $n$-fold differentiation of (1.2) we reach

$$
s J^{(n+1)}(s)+(n+1) J^{(n)}(s)+J^{(n)}(s+1)=0,
$$

which we may rewrite as

$$
\frac{d}{d s}\left\{s^{n+1} J^{(n)}(s)\right\}+s^{n} J^{(n)}(s+1)=0,
$$

both for $\operatorname{Re}(s)>0$. But $J^{(n)}(s)-(-1)^{n} n ! / s^{n+1}$ is analytic in $\operatorname{Re}(s)>-1$.

The following definitions and identities hold for integers $i>0$, except where otherwise stated. Define inductively

$$
\psi_{0}(s)=\frac{1}{s}, \quad \psi_{i}(s)=\frac{1}{s} \int_{0}^{s} \psi_{i-1}(w+1) d w \quad(\operatorname{Re}(s)>-i, i \geqslant 1),
$$

and write

$$
f_{0}(s)=J(s)=\psi_{0}(s)-\psi_{1}(s)+\cdots+(-1)^{i-1} \psi_{i-1}(s)+(-1)^{i} f_{i}(s) .
$$

Note that this implies

$$
f_{i}(s)=\psi_{i}(s)-f_{i+1}(s) .
$$

For $i \geqslant 1$ the equation (1.2) then induces

$$
\frac{d}{d s}\left\{s f_{i}(s)\right\}+f_{i}(s+1)=\psi_{i-1}(s+1)
$$

where $s f_{i}(s) \rightarrow 0$ as $s \rightarrow 0$. Using (2.2), and (1.2), (1.3) if $i=0$, we obtain

$$
s f_{i}(s)+\int_{0}^{s} f_{i}(w+1) d w=s \psi_{i}(s),
$$

which continues $f_{i}(s)$ to $\operatorname{Re}(s)>-i$ (e.g., via continuations to $\operatorname{Re}(s)>-k$, for each $k<i$, by induction on $k$ ). Because of (2.4) this also says

$$
s f_{i+1}(s)=\int_{0}^{s} f_{i}(w+1) d w .
$$


Differentiation of (2.2) gives

$$
\psi \delta^{(n)}(s)=(-1)^{n} n ! / s^{n+1}, \quad s \psi_{i}^{(n+1)}(s)+(n+1) \psi_{i}^{(n)}(s)=\psi_{i-1}^{(n)}(s+1) .
$$

This may be rewritten as

$$
\frac{d}{d s}\left\{s^{n+1} \psi_{i}^{(n)}(s)\right\}=s^{n} \psi_{i-1}^{(n)}(s+1) \quad(i \geqslant 1)
$$

3. Computation of $\psi_{i}^{(n)}(s)$. We restrict our account to the case when $s$ is real and $s>0$, although this restriction (which, together with $n \geqslant 0$, will be implicit throughout this section) could probably be relaxed, if it were necessary.

LEMMA 1. If $i>0, c \leqslant 2, s>0$, then

$$
\int_{0}^{s} \frac{w^{n} d w}{(w+i)^{n+c}} \leqslant \frac{s^{n+1}}{(n+1) i(s+i)^{n+c-1}} .
$$

Use the substitution $1+i / w=1 / y$, for which $i y / w=1-y$. The integral is

$$
\begin{aligned}
\int_{0}^{s} y^{n+c} w^{2-c} d w / w^{2}= & \frac{1}{i} \int_{0}^{s /(s+i)} y^{n+2}(w / y)^{2-c} d y / y^{2} \\
= & \frac{1}{i} \int_{0}^{s /(s+i)}\left(\frac{i}{1-y}\right)^{2-c} y^{n} d y \\
= & \frac{1}{(n+1) i}\left(\frac{s}{s+i}\right)^{n+1}\left(\frac{i}{1-s /(s+i)}\right)^{2-c} \\
& -\frac{2-c}{(n+1) i^{2}} \int_{0}^{s /(s+i)}\left(\frac{i}{1-y}\right)^{3-c} y^{n+1} d y,
\end{aligned}
$$

by integration by parts. The result of the lemma follows.

An ineffective version of the bounds for $\psi_{i}^{(n)}(s)$ and $f_{i}^{(n)}(s)$ contained in the next lemma would follow easily using Cauchy's inequalities for the derivatives of an analytic function. Observe also that when $n>e-1$ Lemma 2 shows

$$
\psi_{i}^{(n)}(s) \rightarrow 0, \quad f_{i}^{(n)}(s) \rightarrow 0 \quad \text { as } i \rightarrow \infty,
$$

by the ratio test.

LEMMA 2. If $y_{i}(s)$ is either $\psi_{i}(s)$ or $f_{i}(s)$, then

$$
0 \leqslant(-1)^{n} y_{i}^{(n)}(s) \leqslant \frac{n !}{i !(n+1)^{i}(s+i)^{n+1-i}}
$$

for $i>0$.

We obtain, from (1.1), (2.3), and (2.2),

$$
0 \leqslant(-1)^{n} f_{0}^{(n)}(s) \leqslant \int_{0}^{\infty} x^{n} e^{-s x} d x=n ! / s^{n+1}=(-1)^{n} \psi\left(\delta^{n)}(s),\right.
$$

so that the result holds when $i=0$. For the cases $i \geqslant 1$ we proceed by induction on i. From (2.7), (2.9) we have in either case

$$
s^{n+1} y_{i}^{(n)}(s)=\int_{0}^{s} w^{n} y_{i-1}^{(n)}(w+1) d w
$$


The left inequality of the lemma follows at once. For the other the inductive hypothesis yields

$$
(-1)^{n} s^{n+1} y_{i}^{(n)}(s) \leqslant \frac{n !}{(i-1) !(n+1)^{i-1}} \int_{0}^{s} \frac{w^{n}}{(w+i)^{n-i+2}} d w,
$$

so that the induction proceeds because of Lemma 1 . This proves Lemma 2.

Our computation of $\psi_{i}^{(n)}(s)$ is based on following the recurrences (2.8). It is clear that the following theorem provides an algorithm for the computation of $\psi_{i}^{(n)}(s)$ to any prescribed degree of accuracy.

THEOREM 1. Fix an integer $N \geqslant 1$. Define $Y_{i, n}(s)$ for $n=N-1, N-2, \ldots, 0$ in turn by the relations

$$
\begin{array}{cc}
(n+1) Y_{i, n}(s)=Y_{i-1, n}(s+1)-s Y_{i, n+1}(s) & (i \geqslant 1), \\
Y_{0, n}(s)=(-1)^{n} n ! / s^{n+1} ; & Y_{i, N}(s)=0 \quad(i \geqslant 1) .
\end{array}
$$

Then, for each $i \geqslant 0, n \geqslant 0, s>0$, we have

$$
Y_{i, n}(s) \rightarrow \psi_{i}^{(n)}(s) \text { as } N \rightarrow \infty
$$

This last relation holds in the effective form (3.3).

For convenience, renormalize from $\psi_{i}^{(n)}(s)$ to $z_{i, n}(s)$, where

$$
(-1)^{n} \psi_{i}^{(n)}(s)=\frac{1}{i !} \frac{n !}{(n+1)^{i}} \cdot \frac{(s+i)^{i}}{(s+i)^{n+1}} \cdot z_{i, n}(s)
$$

with a similar relation connecting $Y$ with $Z$. Then Lemma 2 shows

$$
0 \leqslant z_{i, n}(s) \leqslant 1 \text {. }
$$

The recurrence $(2.8)$ gives

$$
z_{i, n}(s)=\left(\frac{n+1}{n+2}\right)^{i} \frac{s}{s+i} z_{i, n+1}(s)+\frac{i}{s+i} z_{i-1, n}(s+1) .
$$

We may remark that this or a similar renormalization is desirable in an implementation of the algorithm, to avoid explicit handling of numbers of the inconvenient size of $\psi_{0}^{(n)}(s)$.

Write $z=Z+\delta$. Then

$$
\begin{gathered}
\delta_{i, n}(s)=\left(\frac{n+1}{n+2}\right)^{i}\left(\frac{s}{s+i}\right) \delta_{i, n+1}(s)+\frac{i}{s+i} \delta_{i-1, n}(s+1) \\
\delta_{0, n}(s)=0 ; \quad \delta_{i, N}(s)=z_{i, N}(s) \quad(i \geqslant 1)
\end{gathered}
$$

We will establish by induction that

$$
0 \leqslant \delta_{i, n}(s) \leqslant i \frac{n+1}{N+1}\left(\frac{s+i-1}{s+i}\right)^{N-n} \quad(0 \leqslant n \leqslant N, i \geqslant 0) .
$$

When $n=N$ or when $i=0$ this follows using (3.1). When $n<N$ and $i>0$ we may assume (3.3) holds already with $n$ replaced by $n+1$, and also with $i$ replaced 
by $i-1$. We then obtain from (3.2)

$$
\begin{aligned}
0 \leqslant \delta_{i, n}(s) \leqslant & i\left(\frac{n+1}{n+2}\right)^{i} \cdot \frac{s}{s+i} \cdot \frac{n+2}{N+1} \cdot\left(\frac{s+i-1}{s+i}\right)^{N-n-1} \\
& +(i-1) \frac{i}{s+i} \cdot \frac{n+1}{N+1} \cdot\left(\frac{s+i-1}{s+i}\right)^{N-n} \\
& =i \frac{n+1}{N+1}\left(\frac{s+i-1}{s+i}\right)^{N-n}\left\{\frac{s}{s+i-1}\left(\frac{n+1}{n+2}\right)^{i-1}+\frac{i-1}{s+i}\right\} \\
& \leqslant i \frac{n+1}{N+1}\left(\frac{s+i-1}{s+i}\right)^{N-n},
\end{aligned}
$$

as required, because

$$
\frac{s}{s+i-1}+\frac{i-1}{s+i-1}=1 .
$$

This proves (3.3), and the assertion of the theorem follows.

4. Computation of $f_{i}^{(n)}(s)$. Our algorithm for computing these numbers is based upon expressing $f_{i}^{(n)}(s-1)$ as a Taylor series in the numbers $f_{i}^{(n)}(s)$, and then truncating the system of linear equations in these numbers that results from Eq. (2.5) and its derivatives. An almost equivalent procedure would be based similarly on the integrated form (2.6).

The next lemma relates to the system of linear equations that arises in this way.

Lemma 3. Suppose $\lambda_{n} \geqslant 1$, and let $A$ be the $N$ by $N$ upper triangular matrix

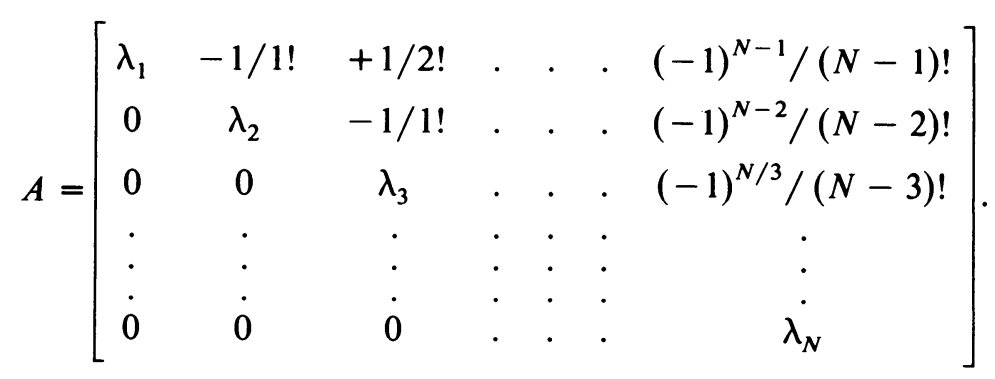

Then the elements of the inverse matrix $A^{-1}=\left(a^{i, j}\right)$ satisfy

$$
\left|a^{i, i+m}\right| \leqslant(1 / \log 2)^{m} \quad(m \geqslant 0) .
$$

For brevity write $1 / \log 2=M$. Consider first the system of equations $A \mathbf{x}=\mathbf{b}$, where $\mathbf{x}$ is the column vector $\left\{x_{1}, \ldots, x_{N}\right\}$ and $\mathbf{b}=\{0, \ldots, 0,1\}$. Then $\left|x_{N}\right| \leqslant 1$. An induction shows $x_{N-n} \leqslant M^{n}$, since, for the inductive step, we obtain

$$
\left|x_{N-n}\right|<\frac{M^{n-1}}{1 !}+\frac{M^{n-2}}{2 !}+\ldots=M^{n}\left\{e^{1 / M}-1\right\}=M^{n}
$$

Now consider the $j$ th column, rows $i$ to $j$, of the matrix product $A A^{-1}$. We see that

$$
x_{1}=a^{i, j}, \quad x_{2}=a^{i+1, j}, \ldots, x_{j-i+1}=a^{j, j}
$$

satisfy a system of linear equations of the type just considered. The result of the lemma follows. 
We can now describe the algorithm for computing the numbers $f_{I}^{(n)}(1)$; we confine our attention to the simplest point 1 that was of importance in [5]. The function $f_{I}$ satisfying (2.5) is analytic in $\operatorname{Re}(s)>-I-1$. Differentiate $n$ times and then set $s=0$. This gives, when $I \geqslant 1$,

$$
f I^{(n)}(1)+(n+1) \sum_{j=0}^{\infty}(-1)^{j} f_{I}^{(n+j)}(1) / j !=\psi(n-1)
$$

For $n \leqslant N$ rewrite these as

$$
\frac{n+2}{n+1} f Y^{n)}(1)+\sum_{1<j<N-n}(-1)^{j} f !^{(n+j)}(1) / j !=\psi\left(Y_{-1}^{()}(1) /(n+1)-\delta_{n, N, I},\right.
$$

where

$$
\delta_{n, N, I}=\sum_{j>N-n}(-1)^{j} f_{I}^{(n+j)}(1) / j !
$$

THEOREM 2. Fix integers $I \geqslant 1, N \geqslant 1$. Let $F_{n}$ denote the solution (obtainable by back-substitution) of the triangular system of linear equations

$$
\frac{n+2}{n+1} F_{n}+\sum_{1<j<N-n}(-1)^{j} F_{n+j} / j !=\psi(n) 1(1) /(n+1) .
$$

Then, for each $n \geqslant 0$,

$$
F_{n}-f l^{n)}(1) \rightarrow 0 \text { as } I \rightarrow \infty .
$$

This relation holds in the effective form (4.2).

Note that the following proof does not in any way suggest that the corresponding property holds as $N \rightarrow \infty$.

The quantities $E_{n}=F_{n}-f_{I}^{(n)}(1)$ satisfy

$$
\frac{n+2}{n+1} E_{n}+\sum_{1<j<N-n}(-1)^{j} E_{n+j} / j !=\delta_{n, N, I} \quad(0 \leqslant n \leqslant N) .
$$

The matrix of this system is of the type considered in Lemma 3. Therefore

$$
\left|E_{n}\right| \leqslant \sum_{0<m<N-n} M^{m} \delta_{n+m, N, I} \leqslant \frac{1}{M^{n}} \sum_{t<N} M^{t} \delta_{t, N, I},
$$

where we write $M=1 / \log 2$, as before.

In (4.1) we use the estimate provided by Lemma 2 . This gives

$$
\left|E_{n}\right| \leqslant \frac{1}{M^{n}} \frac{(I+1)^{I-1}}{I !} \sum_{\substack{t<N \\ j>N-t}} \frac{M^{t}(t+j) !}{j !(t+j+1)^{I}(I+1)^{t+j}} .
$$

Writing $t=N+1-u, t+j=N+v$, we obtain

$$
\left|E_{n}\right| \leqslant \frac{(I+1)^{I-1}}{I !} \frac{M^{N-n+1}}{(N+2)^{I}} \sum_{v>1} \frac{N !}{(I+1)^{N+v}} \frac{(N+v) !}{N ! v !} \sum_{u>1} \frac{v !}{(u+v-1) ! M^{u}} .
$$

The inner sum over $u$ does not exceed

$$
\sum_{u>1} \frac{1}{u ! M^{u}}=-1+e^{1 / M}=1
$$


since $M=1 / \log 2$. Therefore the sum over $v$ does not exceed

$$
\frac{N !}{(I+1)^{N}}\left(1-\frac{1}{I+1}\right)^{-(N+1)}=\frac{N !(I+1)}{I^{N+1}} .
$$

Consequently

$$
\left|E_{n}\right| \leqslant \frac{1}{M^{n}} \frac{N !}{I !}\left(\frac{I+1}{N+2}\right)^{I}\left(\frac{M}{I}\right)^{N+1} .
$$

The qualitative assertion of the theorem now follows by the ratio test, since $N+2 \geqslant 3>e$.

We may also observe from (4.2) that the choice $N=I$, although not necessarily the best, is a good one, since in that case

$$
\left|E_{n}\right| \leqslant \frac{1}{M^{n}}\left(\frac{M}{I}\right)^{I+1},
$$

which is very good for large $I$.

5. Numerical Considerations. The proof of Theorem 1 also shows that the algorithm it describes does not involve undesirable propagation of machineinduced rounding errors. This is because the algorithm consists of following the recurrences (2.8) for $n \leqslant N$, in the stable direction of decreasing $n$, where for $i \geqslant 1$ the quantity $\psi_{i}^{(N)}(s)$ has been replaced by the incorrect value 0 . The method of proof shows that if the renormalized quantities $z_{i, N}(s)$ are affected by any errors $\Delta_{i, N}(s)$ satisfying $\left|\Delta_{i, N}(s)\right| \leqslant 1$ then the resulting error in $z_{i, n}(s)$ is bounded by the expression on the right of (3.3). In view of (3.1) and the linearity of the recurrences (2.8) this shows that errors in $z_{i, m}(s)$ of relative (and hence absolute) magnitude not exceeding $\varepsilon$, say, induce (in the first place) smaller errors in $z_{i, m-1}(s)$ and (eventually) errors in $z_{i, n}(s)$ which, even after summation over $m$, do not exceed

$$
\varepsilon i \sum_{m>n}\left(1-\frac{1}{s+i}\right)^{m-n}=\varepsilon i(s+i)
$$

and this only on pessimistic assumptions about the way in which separate machine-induced errors might combine.

We can also show that serious loss of significant digits due to cancellation does not occur in the algorithm described by Theorem 2, provided we do not choose $N$ too large in relation to $I$. For the quantity $F_{n}$ is expressible as

$$
F_{n}=\sum_{n+m<N} a^{n, n+m} \psi Y_{-1}^{n+m)}(1) /(n+m),
$$

with $\left|a^{n, n+m}\right| \leqslant M^{m}(M=1 / \log 2)$ as in Lemma 3. If each term in this sum were evaluated with a relative error not exceeding $\varepsilon$, then use of Lemma 2 guarantees that the absolute error in the result would not exceed

$$
\frac{\varepsilon}{M^{n}} \frac{I^{I}}{I !} \sum_{m<N-n} \frac{(n+m) !}{(n+m+1)^{I-1}}\left(\frac{M}{I}\right)^{n+m} .
$$

For maximum control of this expression we might specify $N \leqslant I / M$, to ensure that the terms in the sum decrease as $m$ increases. 
Similar considerations indicate that, having computed $\psi$ via Theorem 1 and $f$ via Theorem 2, loss of significance does not occur in obtaining $J^{(n)}(1)$ via (2.3). For the estimate in Lemma 2 for $\psi_{i}^{(n)}(1)$ does not exceed

$$
n ! \frac{(i+1)^{i+1}}{(i+1) !} \frac{1}{(n+1)^{i}(i+1)^{n+1}} \leqslant \frac{n ! e^{i}}{(n+1)^{i}(i+1)^{n}}
$$

which for $n \geqslant 2$ decreases rapidly with increasing $i$. This estimate is not very helpful when $n=0$ or 1 , but the numerical evidence indicates that $\psi_{i}^{(n)}(1)$ decreases with $i$ for each $n$.

A numerical experiment, of performing all computations to a restricted accuracy, indicates that this method of computing $J^{(n)}(1)$ gives answers accurate to within a few units of the last significant digit retained by the machine employed.

We may measure the speed of convergence of the algorithm by asking that the estimate (4.2) of the truncation errors should not exceed $\varepsilon$, and then counting how many times the recurrence relation in Theorem 1 is employed, since this is where the bulk of the work lies. Choosing $N=N_{2}=I$ in Theorem 2 for simplicity, we require

$$
\left(\frac{M}{I}\right)^{I+1}<\varepsilon
$$

We must also compute $\psi_{i}^{(n)}(1)$ for $i \leqslant I, n \leqslant N$, to the same relative accuracy $\varepsilon$, say. For each $i$ this is accomplished by one application of Theorem 1, where because of (3.1) and (3.3) we may demand

$$
\left(1-\frac{1}{i+1}\right)^{N_{1}-N_{2}}<\frac{\varepsilon}{i}
$$

This says $\left(N_{1}-N_{2}\right) \log (1+1 / i)<\log (i / \varepsilon)$, so that

$$
N_{1}>N_{2}+i \log (i / \varepsilon)
$$

is sufficient. This would involve $i N_{1}$ references to the recurrence in Theorem 1 . Consequently the amount of work involved is asymptotic (as $\varepsilon \rightarrow 0)$ to

$$
\sum_{i<I} i^{2} \log (1 / \varepsilon) \sim \frac{1}{3} I^{3} \log (1 / \varepsilon)
$$

Since $I>\log (1 / \varepsilon)$ is then sufficient to secure (5.1), for small $\varepsilon$, the work needed is at most $O\left\{\log ^{4}(1 / \varepsilon)\right\}$. In this respect our algorithm compares very favorably with, say, popular algorithms for one-dimensional numerical quadrature.

To obtain the data in Tables 1 and 2 , we computed $\psi_{i}^{(n)}(1)$ for $0 \leqslant n \leqslant 10$, $1 \leqslant i \leqslant 15$ by the algorithm of Theorem 1 , with $N=N_{1}$ chosen in accordance with (5.2). Then we computed $f_{I}^{(n)}(1)$ via Theorem 2 for $1 \leqslant I \leqslant 15$, where we took $N=N_{2}=I$. In (5.2) we chose $\varepsilon=2^{-128}$; the ICL 2980 computer used was instructed to allocate 128 bits per machine real number. The numbers $J^{(n)}(1)$ were then obtained from (2.3). With this choice of parameters the error bound (4.3) only guarantees an accuracy of $\pm 10^{-17}$ in Table 1 , but the observed rate of convergence of the approximations as $I$ increases (illustrated in Table 2) indicates that the tabulated results are accurate to the number of figures quoted. 
TABLE 1

\begin{tabular}{|r|c|}
\hline$n$ & $(-1)^{n} J^{(n)}(1) / n !$ \\
\hline 0 & 0.6243299885435508709929363 \\
1 & 0.8533915293192851280027471 \\
2 & 0.9408920196735678871087327 \\
3 & 0.9755064320848035533150307 \\
4 & 0.9896144672153733387131122 \\
5 & 0.9955113408165600524664809 \\
6 & 0.9980287948084816308130048 \\
7 & 0.9991227235350166334484403 \\
8 & 0.9996051772985864668017361 \\
9 & 0.9998206201345428307349949 \\
10 & 0.9999178444065401486364825 \\
\hline
\end{tabular}

It is not suggesed that one would in fact allocate numerical storage to the numbers $J^{(n)}(1)$ for large $n$, since (2.3) and Lemma 2 show

$$
(-1)^{n} J^{(n)}(1) / n ! \rightarrow 1 \text { as } n \rightarrow \infty .
$$

In fact, in the application in [5] the numbers worthy of storage were $\psi_{2}^{(n)}(1)$ and $f_{3}^{(n)}(1)$.

To illustrate the empirically observed rate of convergence of the algorithm of Theorem 2, Table 2 shows the successive approximations $J_{I}$ to the number $J(1)$, obtained in the manner described for $1 \leqslant I \leqslant 15$. In scanning this table, it should be borne in mind that (working to a fixed precision throughout) the work involved in calculating the $I$ th approximation is asymptotic to $I^{2}$.

TABLE 2

\begin{tabular}{|r|l|}
\hline$I$ & \multicolumn{1}{|c|}{$J_{I}$} \\
\hline 1 & $0.6 \ldots$ \\
2 & $0.624 \ldots$ \\
3 & $0.62432 \ldots$ \\
4 & $0.624329 \ldots$ \\
5 & $0.62432998 \ldots$ \\
6 & $0.6243299885 \ldots$ \\
7 & $0.624329988543 \ldots$ \\
8 & $0.62432998854355 \ldots$ \\
9 & $0.6243299885435508 \ldots$ \\
10 & $0.624329988543550870 \ldots$ \\
11 & $0.62432998854355087099 \ldots$ \\
12 & $0.62432998854355087099293 \ldots$ \\
13 & $0.6243299885435508700929363 \ldots$ \\
14 & $0.6243299885435508709929363 \ldots$ \\
15 & $0.6243299885435508709929363 \ldots$ \\
\hline
\end{tabular}

The calculations described in these tables occupied about 20 seconds of machine time. 
6. Coda. Let $g(n)$ denote the largest prime factor of $n$. Dickman [4] gave a heuristic treatment of the fact and de Bruijn [3] proved that

$$
\lim _{x \rightarrow \infty}\left\{\sum_{n<x} \log g(n) / \sum_{n<x} \log n\right\}=A
$$

where

$$
A=\int_{0}^{\infty} \frac{\rho(x) d x}{(1+x)^{2}},
$$

and Dickman's function $\rho$, continuous in $(0, \infty)$, satisfies

$$
\begin{aligned}
\rho(x) & =1 & & (0 \leqslant x \leqslant 1), \\
x \rho^{\prime}(x) & =-\rho(x-1) & & (x>1), \\
\rho(x) & =O\left(e^{-x}\right) & & (x>1) .
\end{aligned}
$$

Dickman obtained a numerical approximation to $A$ directly from (6.1), using successive numerical integrations of (6.2) to obtain approximations to $\rho$ over successive intervals $(n, n+1)$.

We can show that $A=J(1)$, where $J(1)$ is as in (1.1). Introduce the Laplace transform of $\rho^{\prime}$ :

$$
r(s)=\int_{1}^{\infty} \rho^{\prime}(x) e^{-s x} d x .
$$

In the first place we obtain, using (6.2),

$$
A=\int_{1}^{\infty} \frac{\rho(x-1)}{x^{2}} d x=-\int_{1}^{\infty} \frac{\rho^{\prime}(x)}{x} d x=-\int_{0}^{\infty} r(s) d s .
$$

On the other hand we have

$$
\begin{aligned}
\rho^{\prime}(x) & =0 & & (0<x<1), \\
-x \rho^{\prime \prime}(x) & =\rho^{\prime}(x)+\rho^{\prime}(x-1) & & (x>1),
\end{aligned}
$$

and $\rho^{\prime}$ is continuous in $(1, \infty)$, with $\rho^{\prime}(1+)=-1$. Hence

$$
\begin{aligned}
\frac{d}{d s}\left\{e^{-s}+s r(s)\right\} & =\frac{d}{d s} \int_{1}^{\infty} \rho^{\prime \prime}(x) e^{-s x} d x \\
& =\int_{1}^{\infty}\left\{\rho^{\prime}(x)+\rho^{\prime}(x-1)\right\} e^{-s x} d x=\left(1+e^{-s}\right) r(s),
\end{aligned}
$$

so that

$$
s r^{\prime}(s)-e^{-s} r(s)-e^{-s}=0
$$

This gives

$$
r(s)=\exp \left\{-\int_{s}^{\infty} \frac{e^{-t}}{t} d t\right\}-1
$$

because $r(s) \rightarrow 0$ as $s \rightarrow \infty$. Hence, and by integrations by parts from (6.3),

$$
A=\int_{0}^{\infty} x \frac{d}{d x}\left[\exp \left\{-\int_{x}^{\infty} \frac{e^{-t}}{t} d t\right\}-1\right] d x=J(1),
$$

the constant defined in (1.1).

Department of Pure Mathematics

University College, Cardiff

P. O. Box 78

Cardiff CF1 1XL Wales, Great Britain 
1. R. Bellman \& K. L. Cooke, Differential-Difference Equations, Academic Press, London, 1963.

2. N. G. DE BRUIIN, "On the number of uncancelled elements in the sieve of Eratosthenes," Nederl. Akad. Wetensch. Proc., v. 53, 1950, pp. 803-812 = Indag. Math., v. 12, 1950, pp. 247-256.

3. N. G. DE BRUIIN, "On the number of positive integers $<x$ and free of prime factors $>y$," Nederl. Akad. Wetensch. Proc. Ser. A, v. 54, 1951, pp. 50-60.

4. K. Dickman, "On the frequency of numbers containing prime factors of a certain relative magnitude," Ark. Mat., v. 22A, 1930, pp. 1-14.

5. G. Greaves, “A weighted sieve of Brun's type,” Acta Arith., v. 40, 1981, pp. 301-335.

6. H. Iwaniec, “Rosser's Sieve,” Acta Arith., v. 36, 1980, pp. 171-202.

7. H. IwANIEC, J. VAN DER LUNE \& H. J. J. TE RIELE, "The limits of Buchstab's iteration sieve," Nederl. Akad. Wetensch. Proc. Ser. A, v. 83, 1980, pp. 409-417. 